\title{
Masked presentations of dissociative disorder among Sri Lankan adolescents
}

\author{
M Chandradasa, WKTR Fernando, KALA Kuruppuarachchi
}

\section{Abstract}

Dissociation could occur in adolescence in response to traumatic life experiences disrupting essential areas of psychological functioning. We report three complex presentations of dissociation in adolescents in Sri Lanka.

A 16-year-old girl presented with poor sleep, overtalkativeness and euphoric mood after her father had left home a day before.

During the war, a 14-year-old boy was brought to hospital as he was speaking in Tamil and mentioning the names of terrorist leaders. Later it was found that he is from a Sinhalese family and had experienced violence at home.
A 16-year-old boy presented with irritability, disinhibition and regressed behaviour. A detailed assessment revealed an underlying depressive state in the context of family conflicts.

All the above patients presented with features suggestive of mania, misleading clinicians and leading to unsuccessful treatment strategies. Therefore, in-depth psychosocial assessment is needed to diagnose dissociation in adolescents and to plan evidence-based treatment.

Key words: Dissociative disorders, adolescence, Sri Lanka,

SLJ Psychiatry 2019; 10(1): 25-27

\section{Introduction}

Dissociative disorders are thought to be caused by a discontinuity in the integration of consciousness, identity, emotion, motor control, memory, perception, and behaviour. Dissociation could occur in adolescence in response to traumatic and overwhelming life experiences that disrupt essential areas of psychological functioning (1). Dissociation may occur in the presence of many psychiatric disorders.

Lyssenko et. al., in their meta analysis reported that the most substantial dissociation scores are seen with dissociative disorders, followed by post-traumatic stress disorder, borderline personality disorder, and conversion disorder (2). Furthermore, the same meta-analysis reported a lower range of scores associated with substance-related disorders, eating disorders, schizophrenia, anxiety disorder, obsessive-compulsive disorder, and affective disorders (2). Dissociative symptoms in other psychiatric disorders are of high relevance to the clinician, as they are linked to maladaptive functioning and poorer response to psychotherapeutic interventions $(3,4)$. Dissociation has a negative impact on treatment outcomes, as the subjects dissociate in response to the negative emotions arising in therapy. Furthermore, they may have an insecure attachment pattern that would weaken the therapeutic relationship (4).

The clinical presentation of dissociation is often sophisticated and can mislead clinicians. Due to the limitations in child and adolescent mental health services in Sri Lanka, many adolescents with such presentations may not receive comprehensive psychological assessments $(5,6)$. In the context of these resource-limitations, it is essential to highlight masked presentations of dissociation in Sri Lankan adolescents that may help other mental health professionals. The following descriptions are of three adolescents with complex mental health presentations seen at the Colombo North Teaching Hospital, that led to diagnostic dilemmas and management conundrums.

\section{Case Reports}

The first report is of a 16-year-old girl. She presented to an outpatient psychiatric service with a reduced need for sleep, overtalkativeness and euphoric mood for a 
two-week period. After the initial specialised psychiatric evaluation, her presentation was considered to be a manic episode and she had been commenced on antipsychotics. This management approach did not lead to any improvement in her clinical status, and she was brought to hospital by her family, for further assessment. According to her family, she had been emotionally more attached to her father since childhood, and he had left home a day before the onset of the symptoms, after an intense argument with her mother. Antipsychotics were discontinued, and several solution-focused brief family therapy sessions were held with both her parents. Later her symptoms wholly resolved, and she remained psychologically stable after her father returned home.

The second report is of a 14-year-old boy who presented to services during the time of the recent ethnic conflict. The law enforcement authorities had been informed about him, after he was found by a group of villagers. He apparently spoke in Tamil and mentioned names of several terrorist leaders. Many villagers were suspicious of him as this happened at the height of the armed conflict in Sri Lanka and there was a constant threat of deadly bombing by the militants. His behaviour suggested that he did not comprehend Sinhalese. He also exhibited manic-like symptoms with euphoric mood, overtalkativeness, physical over-activity, grandiose ideas and reduced need for sleep for more than a month. There was no existence of multiple identities. Later social investigations revealed that he was from a Sinhalese family, and that he had witnessed violence towards him and his mother at home, prior to the change of behaviour. Treatment with an abreaction, fluoxetine, and traumafocused cognitive behaviour interventions helped him to recover.

The third report is of a 16-year-old boy who presented with irritability, disinhibition, reduced need for sleep, overactivity and regressed behaviour. He had been seen by several psychiatrists in outpatient settings, had been diagnosed as having a manic episode and had been commenced on antipsychotics. A detailed psychological assessment revealed an underlying depressive state with anhedonia, reduced energy and thoughts of hopelessness and guilt. Collateral information from family members revealed that there had been intense conflicts between his older brother and father. He was treated with an abreaction, fluoxetine, and trauma-focused cognitive behaviour interventions, which lead to a stable psychological status and recovery.

All the above adolescents were diagnosed with unspecified dissociative disorder according to the $5^{\text {th }}$ edition of the Diagnostic and Statistical Manual of Mental Disorders, by a consultant psychiatrist (1). Informed written consent was obtained from the parents and adolescents for assessments and publication.

\section{Discussion}

The above case reports describe three Sri Lankan adolescents who developed manic-like presentations in the context of intense psychological conflicts. Treatment as for juvenile bipolar affective disorder in outpatient settings did not facilitate recovery, and they were further assessed at the Colombo North Teaching Hospital. Comprehensive psychological formulations, taking into consideration psycho-social stressors, helped to diagnose masked dissociative symptoms and subsequent targeted management, leading to clinical recovery.

European studies have reported that conflictual relationships between parents could be associated with dissociative symptoms in young people (7). In all three case reports, there were family conflicts before the onset of the symptoms. It is imperative that relevant social investigations, and collection of information from parents and family are carried out when assessing children and adolescents with possible dissociative symptoms.

There have been limited reports of the foreign accent syndrome, which is a rare speech disorder. This is characterised by the appearance of a new accent, different from the speaker's native language and perceived as foreign by others (8). Even though Tamil is not a foreign language, it was a new language for the second adolescent reported. In most patients, this presentation is secondary to focal brain damage. However, it has been reported in association with psychiatric disorders, including dissociative disorders as in the 14-year-old boy we reported (8).

In abreaction, a clinician interviews an individual under the influence of a drug. It could be more precisely described as a technique of emotional catharsis conducted during a drug interview (9). The reported second and third adolescents were treated using this method. According to available systematic reviews, the evidence for the effectiveness of drug interviews is of poor quality (9). However, it may be of benefit in the treatment of the acute dissociative disorder as shown in this report with clinical recovery in the adolescents.

In conclusion, the three case reports demonstrate complex presentations of dissociation in adolescents. All patients presented with a picture suggestive of a manic episode, misleading clinicians and initially leading to unsuccessful treatment strategies. Therefore, a detailed, in-depth psycho-social assessment is needed to diagnose dissociation in adolescents and to plan evidence-based treatment.

\section{Conflicts of interest}

None declared 
M Chandradasa, WKTR Fernando, KALA Kuruppuarachchi, Department of Psychiatry, Faculty of Medicine, University of Kelaniya, Ragama, Sri Lanka and University Psychiatry Unit, Colombo North Teaching Hospital, Ragama, Sri Lanka

Corresponding author: $\mathrm{M}$ Chandradasa

Email: miyuruc@kln.ac.lk

https://orcid.org/0000-0002-1873-8228

\section{References}

1. American Psychiatric Association. Diagnostic and Statistical Manual of Mental Disorders. 5th edn. American Psychiatric Association, Washington, DC, 2013.

2. Lyssenko L, Schmahl C, Bockhacker L, Vonderlin R, Bohus M, Kleindienst N. Dissociation in psychiatric disorders: a meta-analysis of studies using the dissociative experiences scale. Am J Psychiatry 2017; 175(1): 37-46.
3. Haaland VØ, Landrø NI. Pathological dissociation and neuropsychological functioning in borderline personality disorder. Acta Psychiatr Scand 2009; 119(5): 383-92.

4. Spitzer C, Barnow S, Freyberger HJ, Joergen Grabe H. Dissociation predicts symptom-related treatment outcome in short-term inpatient psychotherapy. Aust N Z J Psychiatry 2007; 41(8): 682-7.

5. Chandradasa M, Kuruppuarachchi KA. Child and youth mental health in post-war Sri Lanka. BJ Psych Int 2017; 14 (2): 36-7.

6. Chandradasa M, Champika L. Subspecialisation in Postgraduate Psychiatry and Implications for a ResourceLimited Specialised Child and Adolescent Mental Health Service. Acad Psychiatry 2019; 43(1): 135-39.

7. Bob P, Selesova P, Raboch J, Kukla L. Dissociative symptoms and mother's marital status in young adult population. Medicine (Baltimore). 2015; 94(2): e408.

8. Rajpal N, Chakrabarti S. Dissociative disorder presenting as foreign accent syndrome. World J Psychiatry 2013; 3(4): 103-6.

9. Poole NA, Wuerz A, Agrawal N. Abreaction for conversion disorder: systematic review with meta-analysis. Br J Psychiatry 2010; 197(2): 91-5. 